\title{
Femtosecond laser inscribed advanced calibration phantom for optical coherence tomography (OCT)
}

Lu, Yang, Gordon, Neil, Coldrick, Benjamin, Ibrahim, Izzati, Mezentsev, Vladimir, et al.

Yang Lu, Neil Gordon, Benjamin Coldrick, Izzati Ibrahim, Vladimir Mezentsev, David Robinson, Kate Sugden, "Femtosecond laser inscribed advanced calibration phantom for optical coherence tomography (OCT)," Proc. SPIE 11292, Advanced Fabrication Technologies for Micro/Nano Optics and Photonics XIII, 1129205 (28 February 2020); doi: 10.1117/12.2544310

SPIE. Event: SPIE OPTO, 2020, San Francisco, California, United States 


\title{
Femtosecond laser inscribed advanced calibration phantom for optical coherence tomography (OCT)
}

\author{
Yang Lu*a, Neil Gordon ${ }^{\mathrm{a}}$, Benjamin Coldrick ${ }^{\mathrm{b}}$, Izzati Ibrahim ${ }^{\mathrm{b}}$, Vladimir Mezentsev ${ }^{\mathrm{a}}$, David \\ Robinson $^{\mathrm{c}}$, Kate Sugden ${ }^{\mathrm{a}}$ \\ ${ }^{a}$ Aston Institute of Photonic Technologies, Aston University, Birmingham, B4 7ET, UK;

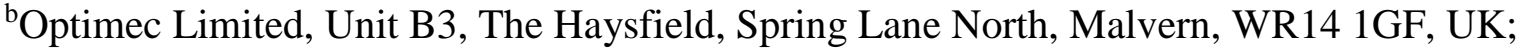 \\ cArden Photonics Ltd., Royston House, 267 Cranmore Boulevard, Solihull, B90 4QT, UK;
}

\begin{abstract}
Optical coherence tomography (OCT) has developed rapidly and is widely used in different fields such as biomedicine and optometry. The characterization and calibration of OCT systems is essential when testing the system and during normal use to ensure that there is no misalignment or distortion that could affect clinical decisions. Imaging distortion is a significant challenge for OCT systems when viewing through non-planar surfaces. Here we present a new multi-purpose plano-convex OCT phantom which is designed to be used for OCT characterization and calibration as well as to validate the post-processing algorithm for the imaging distortion of the OCT systems. A femtosecond laser direct writing technique is used to fabricate this phantom which consists of a landmark layer with radial lines at a 45-degree angular spacing inscribed at $50 \mu \mathrm{m}$ in apparent depth (AD) underneath the planar surface. Below that there are a further 8 layers of a spherical inscription pattern which has a $150 \mu \mathrm{m}$ (in AD) separation between each layer. The first spherical layer is located at $150 \mu \mathrm{m}$ (in $\mathrm{AD}$ ) underneath the planar surface. Due to the laser power loss when travelling through the deeper layer, an increased power is applied to the deeper layers. The spherical pattern overcomes orientation issues seen with existing calibration phantoms. The landmark layer is applied so that it can easily tell the exact location when scanning which will also benefit the image distortion correction process.
\end{abstract}

Keywords: optical coherence tomography (OCT), femtosecond laser inscription, OCT phantom, femtosecond laser direct writing, OCT calibration phantom, OCT image distortion, OCT phantom fabrication

\section{INTRODUCTION}

Optical coherence tomography (OCT) is an imaging technique which can provide a cross-sectional non-invasive real-time in-vivo image by using low-coherence interferometry [1]. OCT has been widely used in the field such as biomedicine and medicine (i.e. ophthalmology) since it was firstly demonstrated by Huang et al. in 1991 [1]. Since then, OCT systems have developed into different types including TD-OCT in 1991 [1], and FD-OCT which has two subsets (SS-OCT and SDOCT) [2] and a growing number of companies have commercialized OCT systems [2]. However, to date, there is no standard approach to be applied in order to calibrate and measure the performance across different systems quantitatively during the installation process and day to day operation. Therefore, a universal multi-purpose phantom which can act as an optical ruler is needed to meet this requirement.

Previously, different methods have been presented to fabricate phantoms such as tissue-mimicking [3] and retinal phantom [4]. However, those methods do not address to all the requirements to quantitatively calibrate and assess the OCT system and associated image distortion when imaging through a curved surface. Femtosecond laser direct writing technique has been used previously for the purpose of phantom inscription at Aston [5], where a non-planar multi-purpose phantom was developed [6] where the planar side could be used for a standard assessment and calibration while the curved side could be used to test the validity of the post-processing algorithm for the image distortion. However, this phantom was inscribed with grid pattern that requires a higher degree of alignment in terms of the angle of the sample to generate a useful calibration phantom for the OCT operator.

*Email: luy12@aston.ac.uk; Phone: +44 1212043616

Advanced Fabrication Technologies for Micro/Nano Optics and Photonics XIII, edited by Georg von Freymann,

Eva Blasco, Debashis Chanda, Proc. of SPIE Vol. 11292, 1129205 · ( 2020 SPIE

CCC code: $0277-786 \mathrm{X} / 20 / \$ 21 \cdot$ doi: $10.1117 / 12.2544310$ 
Here, a new plano-convex OCT phantom is presented to simplify the alignment issues and that includes a landmark layer that can be used to orientate the sample. The phantom can fulfil the purpose of a standard calibration phantom but can also be used to detect distortion and ultimately correct for system distortion through the appropriate use of software algorithms or test the effectiveness of existing algorithms.

\section{EXPERIMENTAL SETUP}

A mode-locked ytterbium-doped femtosecond laser system (Amplitude s-Pulse HP) generated laser pulse which has a central wavelength of $\sim 1026 \mathrm{~nm}$ and a repetition rate range of $1-100 \mathrm{kHz}$. The laser power can be varied from $0 \%$ to $100 \%$ of the total power with the use of an attenuator. The phantom was inscribed at $100 \mathrm{kHz}$ and the laser power was set to be varied from $40 \%$ to $100 \%$ (the average power was $117 \mathrm{~mW}$ for $40 \%$ and $311 \mathrm{~mW}$ for $100 \%$ ). A power meter is positioned below the inscription lens to monitor the output power. This objective lens is from the Mititoyo MPlan Apo NIR series which has a magnification of 100x and a numerical aperture of 0.5 which results of a spot size of $\sim 2 \mu \mathrm{m}$. The laser system is equipped with a high accuracy and high-resolution XY air-bearing stage and a separate mechanical Z-axis with a goniometer mounted on a granite base with which can be software controlled through an Aerotech (A3200) automation controller. The phantom is inscribed on a plano-convex lens (LA4380, Thorlabs) made of fused silica with a refractive index of 1.461. The schematic diagram of the femtosecond laser system set up is shown in Figure 1. The femtosecond laser direct writing technique leads to a permanent refractive index change due to the non-linear multi-photon absorption. The phantom is inscribed through the planar side to minimize aberration effects.

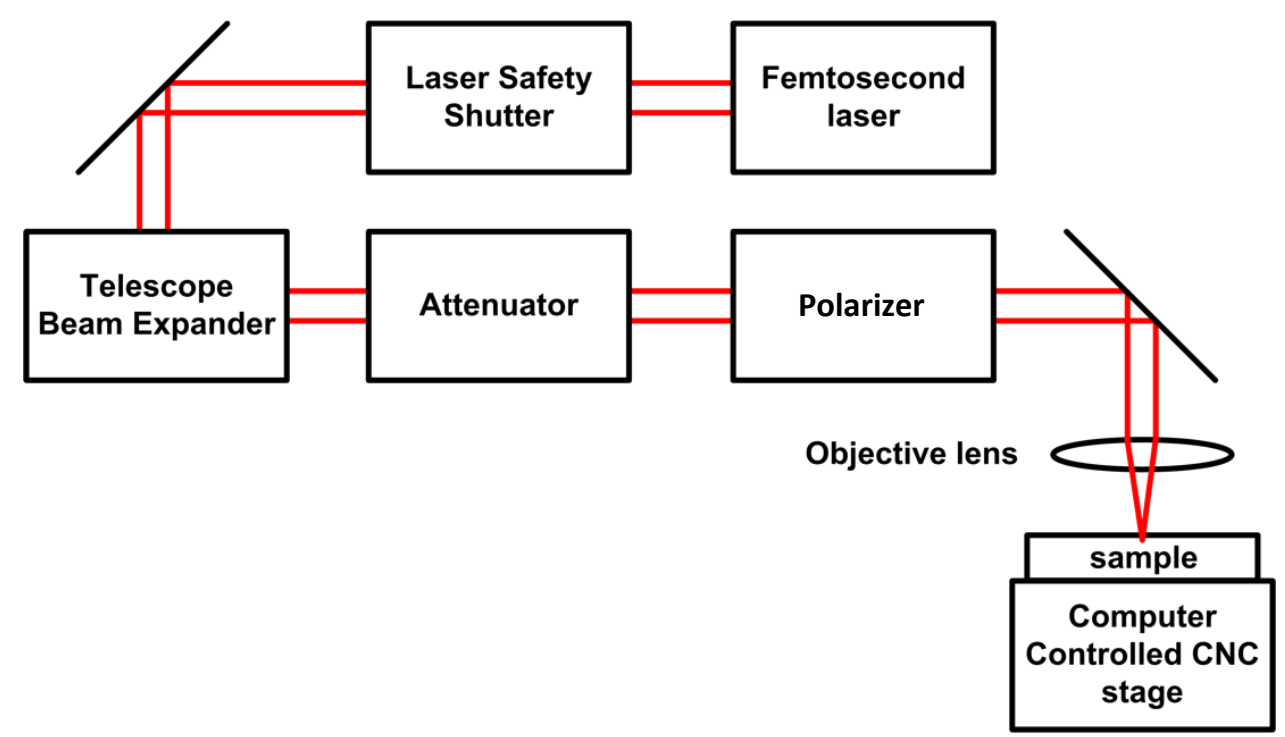

Figure 1. Schematic diagram of the femtosecond laser system setup. The laser has a central wavelength of $1026 \mathrm{~nm}$, the repetition rate can be varied from $1 \mathrm{kHz}$ to $100 \mathrm{kHz}$, the output laser power can be varied from $0 \%$ to $100 \%$ and the objective lens is $100 x$.

The phantom was characterized using an optical microscope (Axioskop 2 MOT Plus, Zeiss) and an OCT system (Optimec is $830 \mathrm{SD}-\mathrm{OCT}$ system). For the microscope measurement, both the $5 \mathrm{x}$ and the 20x objective lens were used to characterize the phantom, where the 5x objective lens was used to capture a top-view pattern image and the 20x objective lens was used to investigate the layer separation and the linewidth data of the phantom. The Optimec is 830 SD-OCT system was used for the OCT characterization. This system is equipped with a super luminescent diode (SLD) light source and a fiber coupler acting as a beam splitter divides the light between the sample and the reference arm [7]. A spectrometer was used to analyze the return reflection combined between the reference arm and the sample scanned by a micro-electrical mechanical (MEMs) mirror system [7]. This OCT system has a central wavelength of 830nm, an axial resolution of $12 \mu \mathrm{m}$ (in air), a lateral resolution of $30 \mu \mathrm{m}$ and a sensitivity of $107 \mathrm{~dB}$ at $1.2 \mathrm{kHz}$, which has a measurement volume of $20 \mathrm{~mm} \mathrm{x}$ $20 \mathrm{~mm}$ x $6 \mathrm{~mm}[7]$. 


\section{PHANTOM DESIGN AND FABRICATION}

The phantom pattern shown in Figure 2 consists of one landmark layer located at $50 \mu \mathrm{m}$ (in apparent depth (AD)) below the surface and 8 layers of the test pattern where the first layer is located at $150 \mu \mathrm{m}$ (in AD) below the surface and each layer has a separation of $150 \mu \mathrm{m}$ so that in total the inscribed depth reaches to $1200 \mu \mathrm{m}$ (in $\mathrm{AD}$, so that the real depth (RD) is $1753.2 \mu \mathrm{m}(1200 \mu \mathrm{m} \times 1.461$ (the refractive index of fused silica) $=1753.2 \mu \mathrm{m})$. A set of radial lines is inscribed in the landmark layer that have an angular separation of 45 degrees. This design enables the end user to easily control the location of the phantom relative to the field of view of the OCT system. With the previous grid pattern, the orientation presented a problem since it affected the separation of the points. To overcome this difficulty, a spherical pattern is inscribed on each layer in this phantom design. Each layer includes a set of 40 circles with increasing radius (i.e. a radius difference of $254 \mu \mathrm{m}$ ), where the radius range varies from $0.254 \mathrm{~mm}$ to $10.16 \mathrm{~mm}$. Due to the power loss when penetrating to deeper layers, each layer is inscribed with different laser powers between $40 \%$ and $100 \%$ of the total laser output power, where $40 \%$ of the laser power was used to inscribe the top layer and $100 \%$ of the laser power was used to inscribe the deepest layer. The laser power difference for each layer is identical, with the aim of obtaining a consistent linewidth for each layer. The pattern was inscribed on a planar-convex fused silica lens which has a central thickness of $3.8 \mathrm{~mm}$ and an edge thickness of $2 \mathrm{~mm}$. The moving speed for the stage was set to $4 \mathrm{~mm} / \mathrm{s}$ and the writing speed was set to $1 \mathrm{~mm} / \mathrm{s}$.

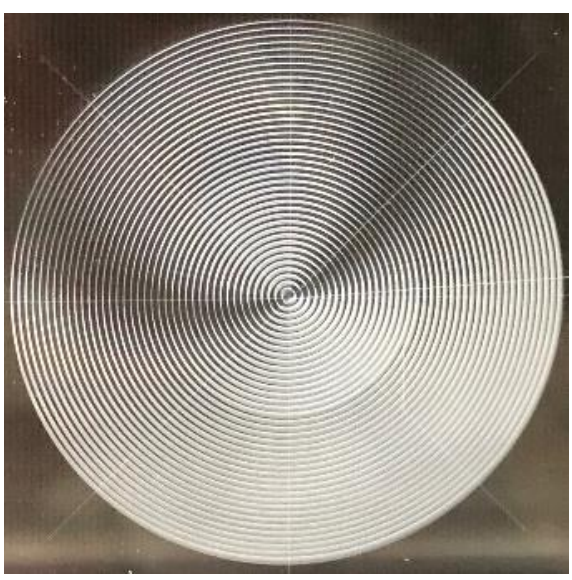

(a)

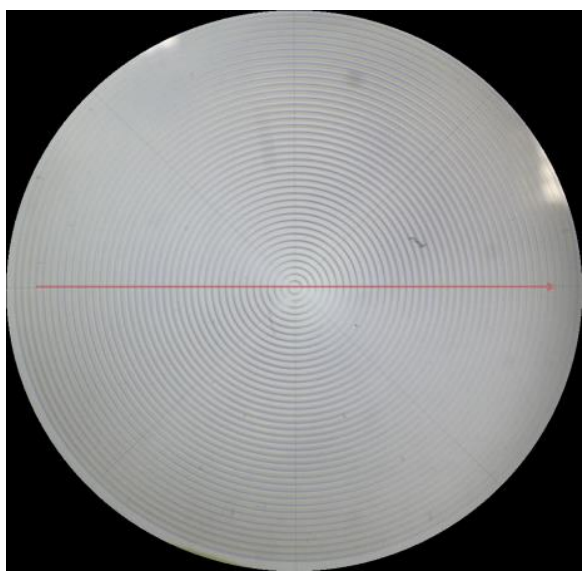

(b)

Figure 2. Phantom design pattern: (a) the pattern initially tested and fabricated on a planar substrate; (b) the pattern fabricated on a curved lens (LA4380, Thorlabs) viewing under an OCT system (Optimec is830 SD-OCT system).

Alignment is always a challenge when placing a sample on the CNC stage to ensure its flatness, especially for a non-planar substrate which increases the difficulty. Four equidistant points on the edge were chosen to assist the alignment. A CCD camera was used in this alignment process to determine the focus position along with the adjustment through both software control of the CNC stage and the manual adjustment of a goniometer base. To ensure a high degree of alignment of the sample, a software program was designed to inscribe various minor marks to verify the surface and the alignment. The alignment is adjusted until at least three points on the edge were focused at the same position, at which point the alignment process is completed and the fourth point can be used to verify the alignment. The program was set to start at the center of the surface. The laser inscription was started from the lowest layer (i.e. the deepest layer) to avoid scattering caused by modified regions.

\section{RESULTS AND DISCUSSIONS}

Both an optical microscope (Axioscope 2 MOT Plus, Zeiss) and an OCT system (Optimec is830) were used to characterize the OCT phantom. The microscope measurement provided information on the linewidth of the inscription in the XY plane which is parallel to the translation stage, and the layer depth for the Z-axis while the OCT system provided a cross-sectional image or a depth profile of the phantom. The comparisons between the setting parameters and the measured parameters of the layer depth are presented in Figure 3 to show the accuracy of the laser fabrication. It can be seen that there is a high 
degree of alignment accuracy so that the measured values are almost identical to the designed value (with $+/-2 \mu \mathrm{m}$ error tolerance) (i.e. the landmark layer was set to $50 \mu \mathrm{m}$ below the planar surface and the measured value of the landmark layer depth was $50.945 \mu \mathrm{m})$.

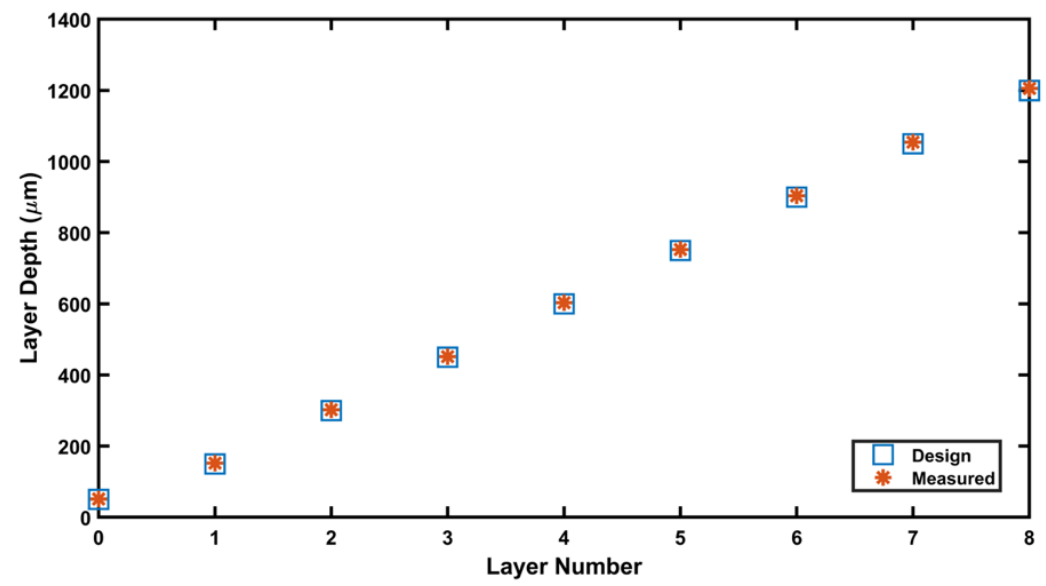

Figure 3. The comparison of design parameter values and measured values from the microscope measurement of the OCT phantom by using a 20x objective lens. The layer depth is in apparent (measured) depth (AD).

The microscope images taken using a 5x objective lens are shown in Figure 4(a) and (b) which Figure 4(a) was taken from a planar surface and Figure 4(b) was taken from a curved surface of the phantom. Figure 4(a) shows that there is high degree of agreement between the design value (i.e. $254 \mu \mathrm{m}$ between each circle) and the measured value for the spherical radius (i.e. $~ 254 \pm 1$ um between each circle). Figure 4(b) shows a microscope image when viewing the phantom from a curved surface. It can be seen that the radius difference between each circle is $\sim 258 \mu \mathrm{m} \pm 1 \mathrm{um}$, which has a $4 \mu \mathrm{m}$ difference to the measurement taken from the planar surface. It may be due to the curvature of the lens that distorted the image slightly, however, the spherical separation remains as $\sim 258 \mu \mathrm{m} \pm 1 \mathrm{um}$ for each circle that can indicate that the sample is not misaligned. Figure 4 (c) and (d) show the linewidth in the XY plane via an optical microscope was measured. The microscope image was acquired using a 20x objective lens taken from the planar side at the 5th layer (at $752.485 \mu \mathrm{m}$ in $\mathrm{AD}$ below the planar surface) showing a linewidth of $6.3 \mu \mathrm{m}$. In Figure 4(d), the microscope image was captured by using a 20x objective lens taken from the planar side at the 7th layer (at $1054.252 \mu \mathrm{m}$ in $\mathrm{AD}$ below the planar surface) showing a linewidth of $6.3 \mu \mathrm{m}$. Therefore, by varying the laser power, the linewidth of the inscription can be consistent for each layer.

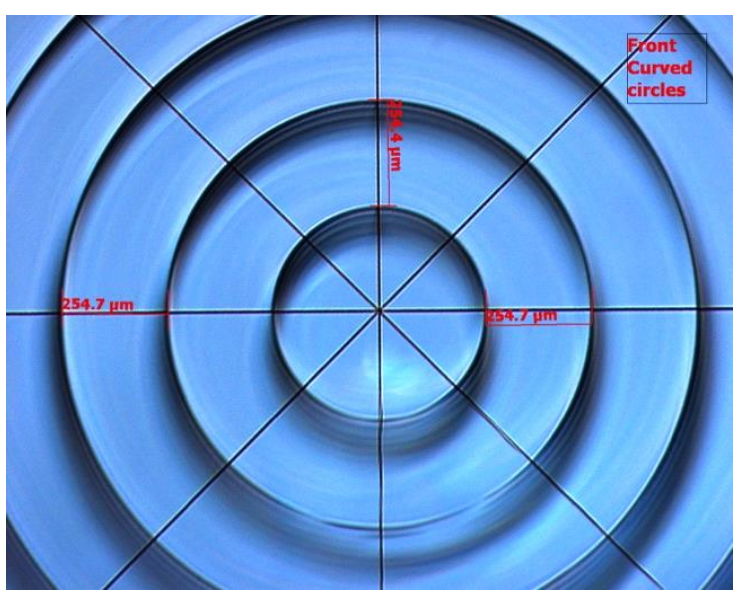

(a)

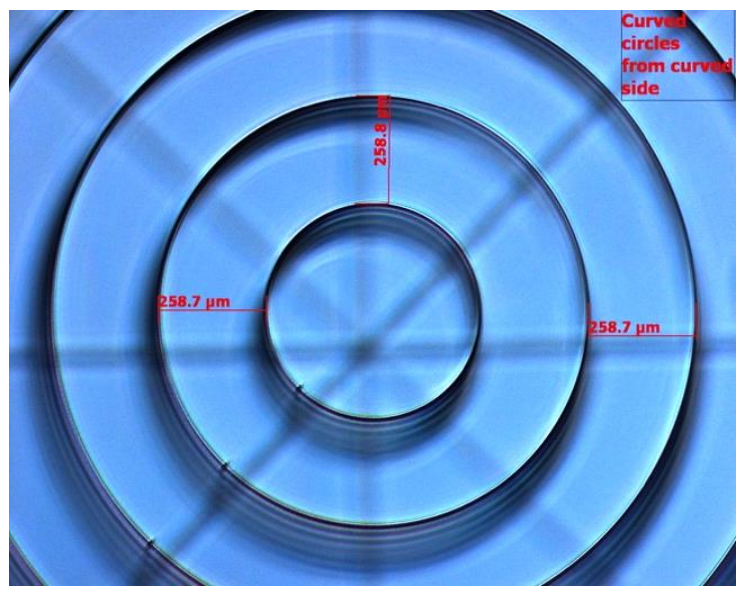

(b) 


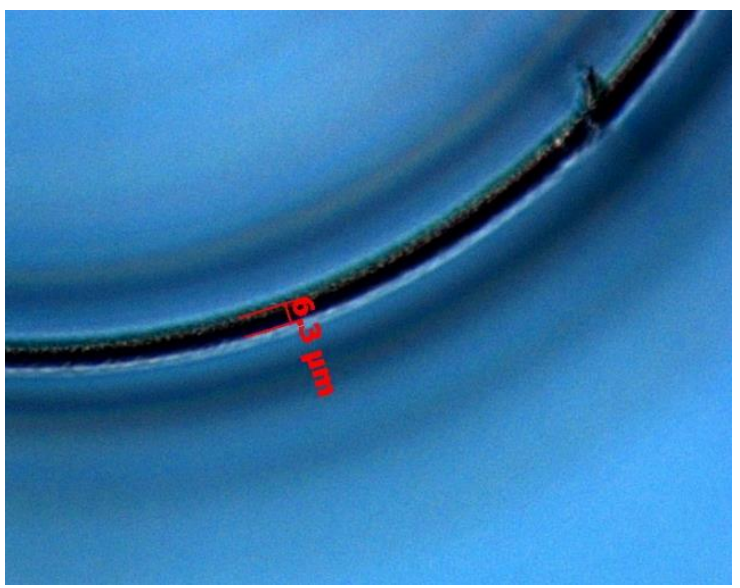

(c)

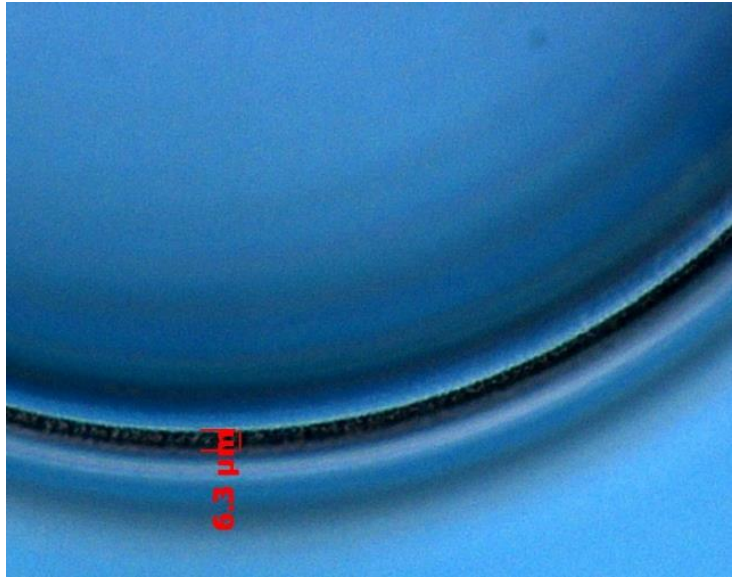

(d)

Figure 4. (a) Microscope image using a 5x objective lens taken from the planar side of the phantom; (b) microscope image using a $5 \mathrm{x}$ objective lens taken from the curved side of the phantom; (c) microscope image using a 20x objective lens taken from the planar side at the 5th layer (at $752.485 \mu \mathrm{m}$ in $\mathrm{AD}$ below the planar surface) showing a linewidth of $6.3 \mu \mathrm{m}$; (d) microscope image using a 20x objective lens taken from the planar side at the 7 th layer (at $1054.252 \mu \mathrm{m}$ in $\mathrm{AD}$ below the planar surface) showing a linewidth of $6.3 \mu \mathrm{m}$.

Subsequent to the microscope characterization, the OCT phantom was then characterized using an OCT system (Optimec is830 system). Example images taken by this system are shown in Figure 5 and Figure 6 that verify the phantom design and illustrate its potential use as a calibration phantom. A solid line for the landmark layer (shown in yellow) indicates that the scan position went through the central point and parallel to a landmark line, this is helpful if using this phantom to correct the image distortion since it gives a known cross-sectional position. Figure 5 shows the view from the planar side of the phantom which is a non-distorted image. It be seen that the landmark layer and all eight layers of the pattern are clearly visible in the OCT image. The parameter measurement on the OCT image matches the designed parameters and the microscope measurements.

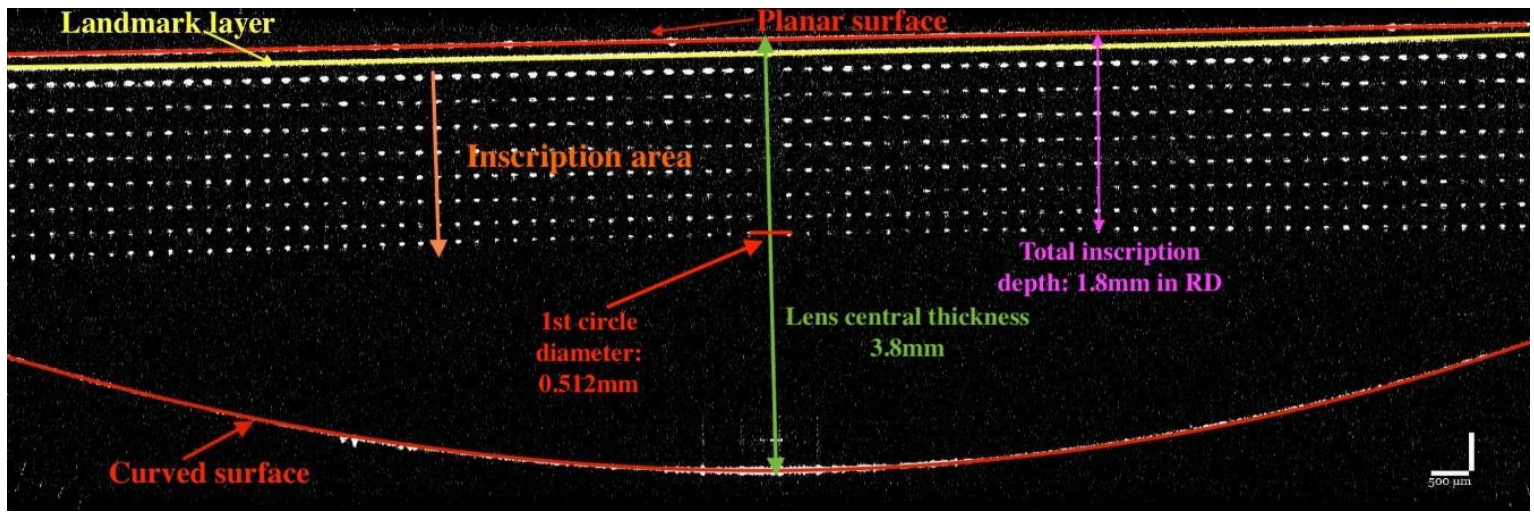

Figure 5. OCT image taken from the planar surface of the phantom. The scan pattern FOV is $19.00 \mathrm{~mm} \times 5.42 \mathrm{~mm}$. The scale bar is $500 \mu \mathrm{m}$. The value indicated on this image is in real depth as the refractive index is applied.

Figure 6 shows an image was taking from the curved surface of the OCT system. The pattern is distorted because of the curvature of the lens. For example, an ophthalmic application measuring a patient's eye, the OCT system would use a postprocessing algorithm to correct the distortion but this assumes a given curvature and refractive index of the lens. Moreover, the central area of this OCT image is much weaker compared with the image taken from the planar surface and the spot seems to be expanded vertically. 


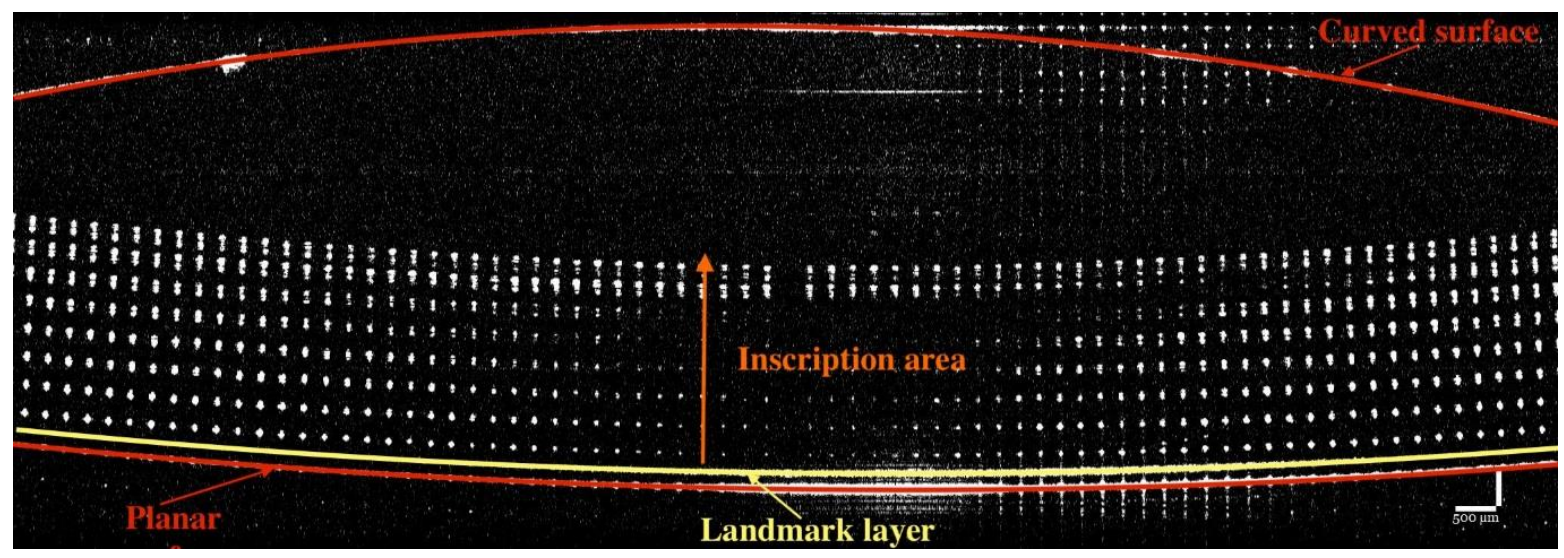

Figure 6. OCT image taken from the curved surface of the phantom. The scan pattern FOV size is $19.00 \mathrm{~mm} \times 5.42 \mathrm{~mm}$. The scale bar is $500 \mu \mathrm{m}$.

\section{CONCLUSIONS}

In summary, for the first time, a spherical pattern with a landmark layer is used in a new phantom design. This phantom can be used to assess, characterize and calibrate the OCT system that are designed to measure non-planar samples. The spherical pattern overcome issues previously observed that were caused by the unknown alignment angle when measuring the phantom with the OCT system. With this new phantom design, the post-processing image distortion correction can be validated since the landmark layer can confirm the exact location and orientation. The planar side can then provide a standard reference when comparing to the curved side. This phantom can be effectively applied to detect and potentially quantitatively correct the distortion based on a self-comparison method. By using the planar side as a standard reference (i.e. non-distorted image) and comparing it with the distorted image, any post-processing algorithms can be validated to ensure optimum system performance.

\section{ACKNOWLEDGEMENTS}

We would like to acknowledge the sponsorship of SPIE for the SPIE Student Chapter Officer Travel Grant and Arden Photonics Ltd. and the School of Engineering and Applied Science at Aston University for the sponsorship of Yang Lu's $\mathrm{PhD}$.

\section{REFERENCES}

[1] Huang, D., Swanson, E. A., Lin, C. P., Schuman, J. S., Stinson, W. G., Chang, W., Hee, M. R., Flotte, T., Gregory, K., Puliafito, C. A. and Fujimoto, J. G., "Optical coherence tomography," Science 254(5035), 1178-1181 (1991).

[2] Fujimoto, J., and Swanson, E., "The Development, Commercialization, and Impact of Optical Coherence Tomography," Investigative ophthalmology \& visual science vol. 57, 9 (2016).

[3] Kennedy, B. F., Loitsch, S., McLaughlin, R. A., Scolaro, L., Rigby, P., and Sampson, D. D., "Fibrin phantom for use in optical coherence tomography," Journal of biomedical optics, 15(3) (2010).

[4] Baxi J., Calhoun, W.R., Sepah, Y.J., Hammer, D.X., Ilev, I., Pfefer, T.J., Nguyen, Q.D., Agrawal, A., "Retinasimulating phantom for optical coherence tomography," Journal of biomedical optics, Sep;19(2) (2013).

[5] Lee, G. C., Rasakanthan, J., Woolliams, P. D., and Sugden, K., "Fabrication of high quality optical coherence tomography (OCT) calibration artefacts using femtosecond inscription," Biophotonics: Photonic Solutions for Better Health Care III , Vol. 8427, International Society for Optics and Photonics (2012).

[6] Lu, Y., Gordon, N., Robinson, D., Coldrick, B., Mezentsev, V., Menduni, F., Fratini, A. and Sugden, K., "Non-planar calibration phantoms for optical coherence tomography," Advanced Fabrication Technologies for Micro/Nano Optics and Photonics XI, Vol. 10544, International Society for Optics and Photonics (2018).

[7] Coldrick, B. J., Richards, C., Sugden, K., Wolffsohn, J. S., and Drew, T. E., "Developments in contact lens measurement: A comparative study of industry standard geometric inspection and optical coherence tomography," Contact Lens and Anterior Eye 39(4), 270-276 (2016). 